\title{
Thin silicon solid-state detectors for energetic particle measurements
}

\author{
Development, characterization, and application on NASA's Parker \\ Solar Probe mission
}

M. E. Wiedenbeck ${ }^{1}$, J. A. Burnham², C. M. S. Cohen ${ }^{2}$, W. R. Cook ${ }^{2}$, R. M. Crabill², A. C. Cummings ${ }^{2}$, A. J. Davis ${ }^{2}$, B. Kecman ${ }^{2}$, A. W. Labrador ${ }^{2}$, R. A. Leske ${ }^{2}$, R. A. Mewaldt ${ }^{2}$, J. S. Rankinn ${ }^{2, \star}$, M. D. Rusert ${ }^{2}$, E. C. Stone ${ }^{2}$, E. R. Christian 3 , P. A. Goodwin 3 , J. T. Link ${ }^{3}$, B. W. Nahory 3 , S. A. Shuman ${ }^{3}$, T. T. von Rosenvinge ${ }^{3}$, C. S. Tindall ${ }^{4}$, H. Black ${ }^{5}$, M. Bullough ${ }^{5}$, N. Clarke ${ }^{5}$, V. Glasson ${ }^{5}$, N. Greenwood ${ }^{5}$, C. Hawkins ${ }^{5}$, T. L. Johnson ${ }^{5}$, A. Newton ${ }^{5}$, K. Richardson ${ }^{5}$, S. Walsh ${ }^{5}$, C. Wilburn ${ }^{5}$, B. Birdwell ${ }^{6}$, D. T. Everett ${ }^{6}$, D. J. McComas ${ }^{7}$, S. E. Weidner ${ }^{7}$,

N. G. Angold ${ }^{8}$, and N. A. Schwadron ${ }^{9}$

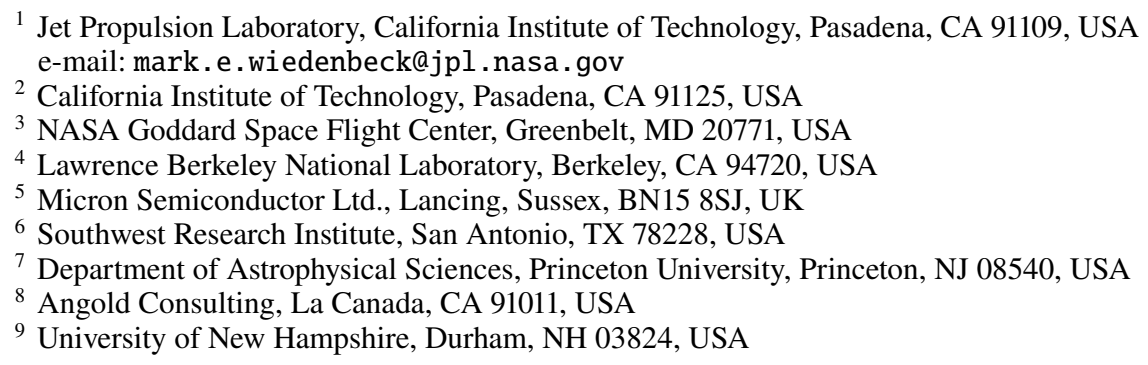

Received 24 October 2020 / Accepted 3 December 2020

\section{ABSTRACT}

\begin{abstract}
Context. Silicon solid-state detectors are commonly used for measuring the specific ionization, $\mathrm{d} E / \mathrm{d} x$, in instruments designed for identifying energetic nuclei using the $\mathrm{d} E / \mathrm{d} x$ versus total energy technique in space and in the laboratory. The energy threshold and species resolution of the technique strongly depend on the thickness and thickness uniformity of these detectors.

Aims. Research has been carried out to develop processes for fabricating detectors that are thinner than $15 \mu \mathrm{m}$, that have a thickness uniformity better than $0.2 \mu \mathrm{m}$ over $\mathrm{cm}^{2}$ areas, and that are rugged enough to survive the acoustic and vibration environments of a spacecraft launch.

Methods. Silicon-on-insulator wafers that have a device layer of the desired detector thickness supported by a thick handle layer were used as starting material. Standard processing techniques were used to fabricate detectors on the device layer, and the underlying handle-layer material was etched away leaving a thin, uniform detector surrounded by a thick, supporting frame.

Results. Detectors as thin as $12 \mu \mathrm{m}$ were fabricated in two laboratories and successfully subjected to environmental and performance tests. Two detector designs were used in the High-energy Energetic Particles Instrument, which is part of the Integrated Science Investigation of the Sun instrument suite on NASA's Parker Solar Probe spacecraft. These detectors have been performing well for more than two years in space.

Conclusions. Thin silicon detectors in $\mathrm{d} E / \mathrm{d} x$ versus total energy instruments enable the identification of nuclei with energies down to $\sim 1 \mathrm{MeV}$ nuc $^{-1}$. This research suggests that detectors at least a factor of two thinner should be achievable using this fabrication technique.
\end{abstract}

Key words. instrumentation: detectors - Sun: particle emission - acceleration of particles - space vehicles: instruments

\section{Introduction}

The measurement of energetic particle populations accelerated by magnetic reconnection in solar flares, by shocks driven by coronal mass ejections, or by shocks or compressions associated with solar wind stream interaction regions is an important tool for understanding these acceleration processes and the transport processes involved in distributing the accelerated particles

\footnotetext{
${ }^{\star}$ Present affiliation: Department of Astrophysical Sciences, Princeton
} University, Princeton, NJ 08540, USA. in the heliosphere. Measurements over a wide range of energies, extending at least from several tens of $\mathrm{keV} \mathrm{nuc}^{-1}$ to several tens of $\mathrm{MeV} \mathrm{nuc}^{-1}$, are needed. This large range is typically covered using two different measurement techniques: time-of-flight $(\mathrm{ToF})$ versus total energy $(E)$ at low energies (e.g., Mason et al. 1998; Hill et al. 2017) and $\mathrm{d} E / \mathrm{d} x$ versus $E$ (e.g., Stone et al. 1998a,b) at high energies. The transition between the two techniques commonly occurs in the vicinity of $1 \mathrm{MeV} \mathrm{nuc}^{-1}$, with some overlap of techniques when possible.

Extending the energy range covered by the $\mathrm{d} E / \mathrm{d} x$ versus $E$ technique to lower energies is important because it will improve 
the energy overlap and, in some applications, allow the energy range of interest to be covered by a single instrument. The main factor limiting the low-energy extension of the $\mathrm{d} E / \mathrm{d} x$ versus $E$ technique is the need for thin, mechanically robust $\mathrm{d} E / \mathrm{d} x$ detectors with a very uniform thickness. In this paper, we discuss the development of such detectors using silicon-on-insulator (SOI) wafers as the starting material. Section 2 discusses the required characteristics of such detectors. Section 3 describes the fabrication process. Section 4 presents data on the thickness characteristics that have been achieved. Section 5 discusses the measured detector performance, including DC electrical characteristics and the response to energetic particles. Section 6 discusses the results of some environmental tests that have been performed. Section 7 briefly discusses possibilities for further extending this technology.

\section{Detector requirements}

In the $\mathrm{d} E / \mathrm{d} x$ versus $E$ technique, an energetic nucleus penetrates a thin detector (the " $\mathrm{d} E / \mathrm{d} x$ " detector), depositing an energy $\Delta E$, and comes to rest in a following thicker detector depositing its residual energy, $E^{\prime}$. The threshold energy for obtaining these two-parameter measurements corresponds to a particle range equal to the sum of the silicon-equivalent thicknesses of the $\mathrm{d} E / \mathrm{d} x$ detector, the dead layer at the front of the $E^{\prime}$ detector, any window in front of the $\mathrm{d} E / \mathrm{d} x$ detector, and the residual range required to reach the energy-detection threshold in the $E^{\prime}$ detector. To penetrate $10 \mu \mathrm{m}$ of silicon, an ion requires an energy of approximately $1 \mathrm{MeV} \mathrm{nuc}^{-1}$, with only a weak dependence on the atomic number, $Z$, of the ion because at these low energies the effective charges of higher- $Z$ elements are reduced by the attachment of orbital electrons from the medium they are traversing. Thus, in order to measure energetic ions down to $\sim 1 \mathrm{MeV}$ nuc $^{-1}$, the $\mathrm{d} E / \mathrm{d} x$ detector must have a thickness of $L \lesssim 10 \mu \mathrm{m}$. Several $\mathrm{d} E / \mathrm{d} x$ versus $E$ instruments that have $\mathrm{d} E / \mathrm{d} x$-detector thicknesses of $\sim 15 \mu \mathrm{m}$ have been used to study heavy-element composition in solar energetic particles (von Rosenvinge et al. 1978, 1995).

Applications requiring even thinner $\mathrm{d} E / \mathrm{d} x$ detectors are particularly challenging because of the difficulty of achieving sufficient thickness uniformity so that the " $\mathrm{d} x$ " involved in obtaining $\mathrm{d} E / \mathrm{d} x$ from the measured energy loss does not excessively degrade the charge and mass resolution. The Voyager Low Energy Charged Particle (LECP) experiment (Krimigis et al. 1977) used some small-area silicon detectors with thicknesses of $\sim 5$ and $\sim 2 \mu \mathrm{m}$ and was able to resolve major elements or element groups and investigate the origin and dynamics of energetic ion populations observed in the magnetospheres of Jupiter (Hamilton et al. 1981) and Saturn (Hamilton et al. 1983).

Further progress in extending the $\mathrm{d} E / \mathrm{d} x$ versus $E$ technique to lower energies depends on the development of detectors that are not only thin but also have a high degree of thickness uniformity. In addition, achieving these characteristics in larger-area detectors is important for a variety of applications.

Stone et al. (1998a) discussed the contributions to mass resolution in $\mathrm{d} E / \mathrm{d} x$ versus $E$ instruments and Mewaldt et al. (2008) applied such analysis to the case of elements and isotopes at a few $\mathrm{MeV} \mathrm{nuc}^{-1}$ in the Low Energy Telescope (LET) instrument on NASA's Solar TErrestrial RElations Observatory (STEREO) spacecraft. This instrument includes relatively thin $(\sim 22-30 \mu \mathrm{m}) \mathrm{d} E / \mathrm{d} x$ detectors. Uncorrectable variations in the thickness of $\mathrm{d} E / \mathrm{d} x$ detectors contribute a relative uncertainty in derived masses that is proportional to the relative uncertainty in the thickness of silicon penetrated, $\sigma_{M} / M \simeq k_{M} \sigma_{L} / L$, where
$k_{M}$ depends on the range-energy relation for the nuclei being measured. Similarly, these variations contribute to the relative charge resolution as $\sigma_{Z} / Z \simeq k_{Z} \sigma_{L} / L$. For the cases discussed here, $k_{M} \simeq 2$ and $k_{Z} \simeq 0.4$.

Thin silicon detectors of the type being discussed in this paper are operating in space in the High-energy Energetic Particles Instrument (EPI-Hi; McComas et al. 2016; Wiedenbeck et al. 2017), which is part of the Integrated Science Investigation of the Sun (IS $\odot$ IS) energetic particle suite (McComas et al. 2016) on NASA's Parker Solar Probe (PSP). This spacecraft was launched on 12 August 2018 on a mission designed to use multiple Venus gravity assists to penetrate deep into the solar corona and investigate the energetic particle environment close to the Sun (Fox et al. 2016).

The sensors in EPI-Hi include two "low-energy telescopes", LET1 and LET2, that were designed to measure heavy ions down to energies $\sim 1 \mathrm{MeV}$ nuc $^{-1}$. Figure 1 is a schematic cutaway illustration of LET1. This double-ended telescope has a pair of thin detectors, LOA (12 $\mu$ m thickness) and L1A (25 $\mu \mathrm{m}$ thickness) at the front of the "A aperture" (top of Fig. 1) and a corresponding pair, $\mathrm{L} 0 \mathrm{~B}$ and $\mathrm{L} 1 \mathrm{~B}$, at the front of the B aperture (bottom of the figure). In this paper we discuss the fabrication, characteristics, and performance of these detectors. The central stack of detectors in LET1 (L2A, L3A, L4A, L4B, $\mathrm{L} 3 \mathrm{~B}$, and L2B) is involved in measuring particles that are sufficiently energetic to fully penetrate beyond both L0 and L1. The high-energy performance of the LET telescopes is discussed elsewhere (McComas et al. 2016; Wiedenbeck et al. 2017).

For measurements of He particles that penetrate LO and stop in $\mathrm{L} 1$, achieving a mass resolution $\sigma_{\mathrm{M}} \simeq 0.25 \mathrm{amu}$ for ${ }^{4} \mathrm{He}$ requires $\sigma_{\mathrm{L}} \simeq 0.4 \mu \mathrm{m}$. This stringent requirement on thickness uniformity was one of the main drivers behind the thin-detector development. This same $\sigma_{\mathrm{L}}$ would lead to a charge resolution $\sigma_{Z} \simeq 0.11$ charge units $(\mathrm{cu})$ for ${ }_{8} \mathrm{O}$ and $\simeq 0.35 \mathrm{cu}$ for ${ }_{26} \mathrm{Fe}$.

Other key requirements for thin detectors are that they be rugged enough to survive the harsh acoustic and vibration environments associated with rocket launches and be capable of stable operation over a wide range of temperatures. In some applications, they must also be able to tolerate high doses of ionizing radiation, including that caused by the energetic nuclei they are being used to detect.

The requirements for thin, uniform detectors that have large areas but are still mechanically robustness are challenging. The following sections discuss the detectors that were developed to meet these requirements.

\section{Detector fabrication}

In order to produce thin, uniform silicon membranes, we use silicon-on-insulator (SOI) wafers purchased from commercial manufacturers as the starting material. A detailed discussion of processes used to make SOI wafers is beyond the scope of this paper, but they have been discussed in a number of reviews (e.g., Gosele \& Tong 1998). In general, one starts with two relatively thick, conventional silicon wafers. A silicon dioxide layer of the desired thickness is grown on one or both wafers and the two wafers are assembled face-to-face with the silicon dioxide layer(s) between them. Applying pressure at elevated temperature leads to the van der Waals bonding of the surfaces. The result is a relatively strong mechanical bond formed without the use of an adhesive.

One wafer, called the "device layer", is used for detector fabrication while the other wafer, called the "handle layer", is used to provide mechanical support. Supported in this way, the device 


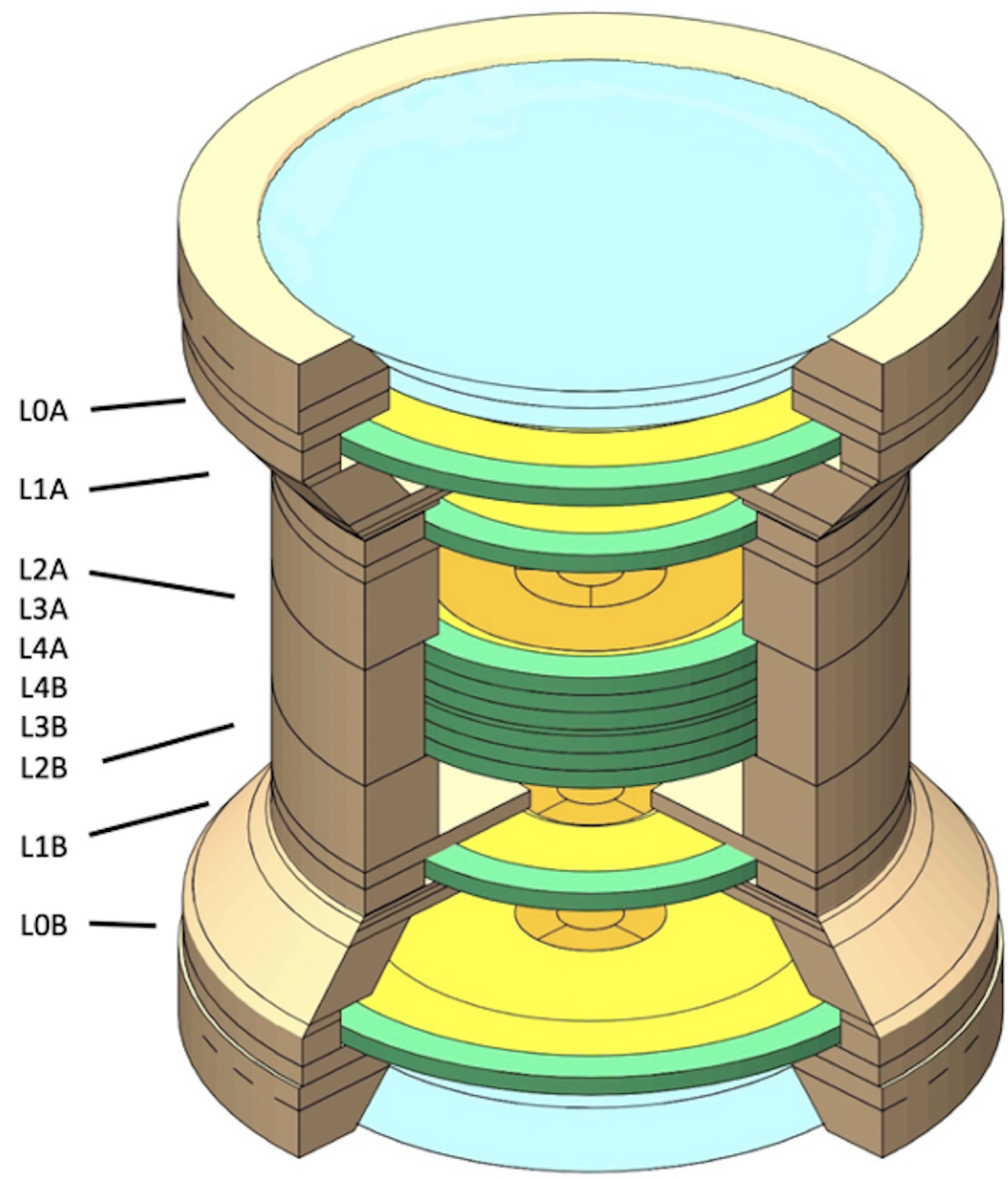

Fig. 1. Schematic cut-away illustration of the LET1 telescope on PSP. Colors indicate different materials, as follows: orangeactive silicon, yellow-inactive silicon, green-polyimide detector mounts, blue-Kapton windows, and brown and beigealuminum telescope housing. The scale can be judged from the fact that the circle encompassing the five active segments of LOB has an area of $1 \mathrm{~cm}^{2}$. The figure was adapted from Wiedenbeck et al. (2017). layer can be thinned by mechanical and/or chemical means to produce a layer that can be thin and, at the same time, very uniform. The thicknesses of the two silicon layers, as well as of the buried-oxide (BOX) layer between them, can be specified by the customer over fairly broad ranges. For the EPI-Hi detectors, thicknesses of $(12 \pm 0.3) \mu \mathrm{m}$ (L0 detectors) or $(25 \pm 0.3) \mu \mathrm{m}$ (L1 detectors), $0.65 \mu \mathrm{m}$, and $500 \mu \mathrm{m}$ were specified for the device, BOX, and handle layers, respectively. We specified the $0.3 \mu \mathrm{m}$ tolerance on the device layer thicknesses on a "best-effort basis" because our specification was tighter than the best tolerance of $0.5 \mu \mathrm{m}$ normally offered by the manufacturer ${ }^{1}$. The Lawrence Berkeley National Laboratory (LBNL) provided the float-zone refined silicon wafers that were used in producing the SOI's device layers because this batch of wafers had previously been shown to yield good conventional detectors.

Figure 2 schematically illustrates a small section of an SOI wafer: (a) as received from the manufacturer and (b) after the processing steps that produce detectors' $p n$ junctions on the outward facing surface of the device layer and etching away the section of the handle layer and the BOX layer that underlie the detector. The handle layer retains its original thickness in regions not under the detectors.

The actual detector fabrication requires numerous steps involving masking, photolithography, ion implantation, annealing, surface passivation, evaporation of aluminum contacts, dicing, installation in mounts, and wire-bonding between pads on the detector chip and on the mount. These steps are routinely carried out during the fabrication of thicker detectors made using conventional silicon wafers and are discussed in various reviews (e.g., Wolf \& Tauber 1998). The essential added step involved

\footnotetext{
1 Ultrasil Corp., Hayward, CA USA.
}

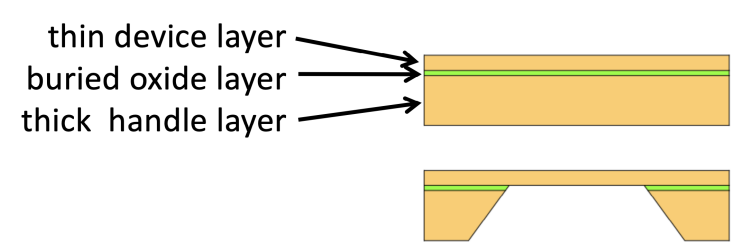

Fig. 2. Schematic illustration (not to scale) of a small section of a 100 or $150 \mathrm{~mm}$ diameter SOI wafer: $(a)$ as received from the manufacturer and $(b)$ after etching steps that remove the parts of the handle layer and buried oxide layers underlying the region that will form the thin detector. For the detectors made for the EPI-Hi instrument, the device, buried oxide, and handle-layer thicknesses were 12 (L0 detector) or $25 \mu \mathrm{m}$ (L1 detector), 0.65 , and $500 \mu \mathrm{m}$, respectively.

in making thin detectors from SOI wafers is the etching away of the handle-layer material. The processes involved in bulk etching of silicon crystals have also been reviewed (e.g., Kovacs et al. 1998).

Our detectors were fabricated from SOI for which singlecrystal silicon wafers having a $\langle 100\rangle$ orientation were used for both the device and handle layers. The etching was done in tetramethyl ammonium hydroxide (TMAH, $\left.\left(\mathrm{CH}_{3}\right)_{4} \mathrm{NOH}\right)$, which has been widely studied and used for bulk etching of silicon (e.g., Tabata et al. 1992). Silicon etches anisotropically in TMAH, with the etch proceeding most rapidly into $\langle 100\rangle$ planes (parallel to the surfaces of our wafers) and most slowly into $\langle 111\rangle$ planes. The etching produces broad, flat-surfaced recesses that terminate at their edges in sides that meet the surface at an angle of $54.74^{\circ}$ $(=\arccos (1 / \sqrt{3}))$. The absolute silicon etching rate and the relative rates for different orientation planes depend on a variety of parameters such as temperature, concentration, amount of 

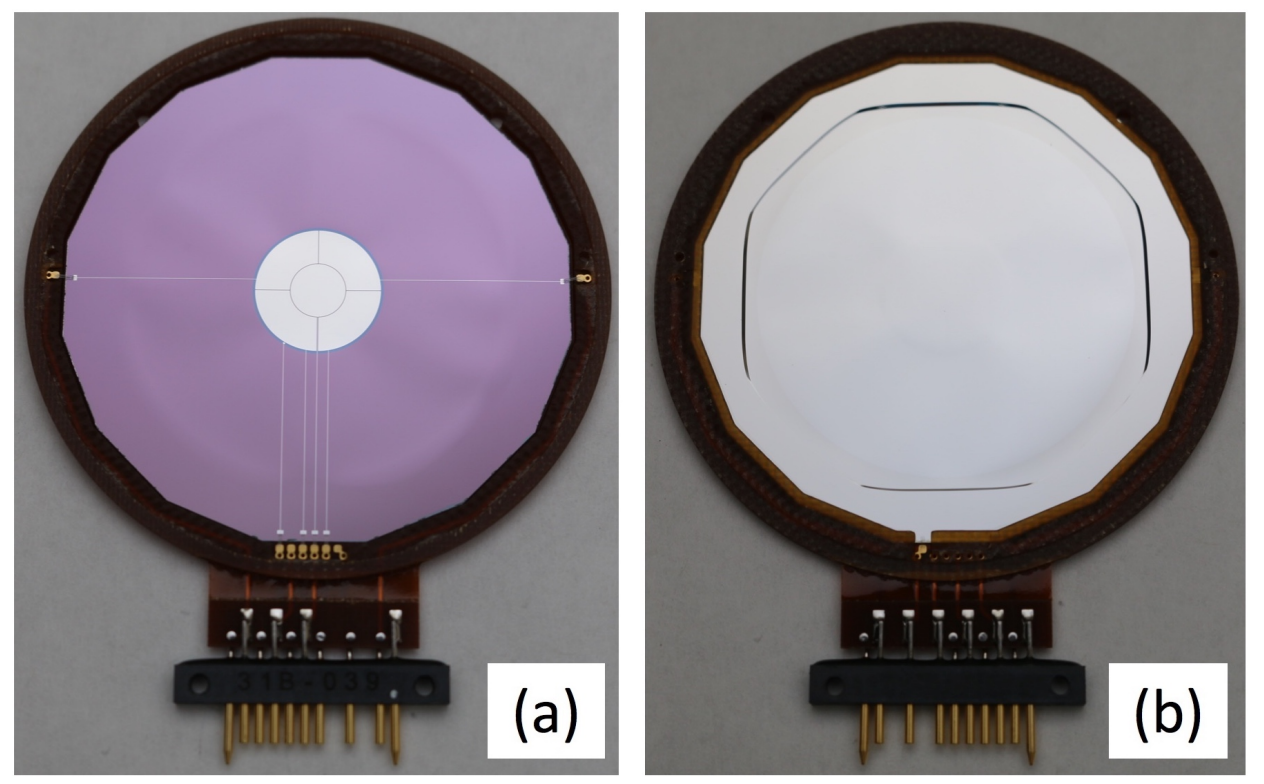

Fig. 3. Photograph of an L0 detector viewed from the junction surface $(a)$ and from the ohmic surface $(b)$. The junction surface is segmented into five active regions, consisting of a central bull's eye surrounded by four quadrants, each with an active area of $0.2 \mathrm{~cm}^{2}$. This 5 -segment structure is located at the center of a silicon membrane of $12 \mu \mathrm{m}$ thickness, the remainder of which is not active. On the ohmic surface, the thin membrane is supported by an approximately octagonal, thick frame, the inner edge of which is visible in the photo. The shape of this edge is due to the different rates at which TMAH etches into different crystal planes. The ohmic surface, including the thin and the thick parts and the transition between them, is metallized with a single aluminum contact.

dissolved $\mathrm{Si}$, and the presence of bubbles (which can locally interfere with the TMAH's contact with the silicon). Producing silicon membranes of uniform thickness depends on the presence of the SOI wafer's BOX layer. The etch rate for that layer's silicon dioxide is much slower (by a factor of 100 or more) than for the silicon (e.g., Tabata et al. 1992). Thus the BOX layer provides an effective etch-stop. When the etching has reached the BOX layer, the SOI is removed from the etchant and inspected to make sure that there are no remaining hillocks of silicon on the BOX and that the BOX has not been penetrated. At that point, a short follow-up etch in hydrofluoric acid (HF) removes the exposed BOX material leaving a smooth silicon surface exposed through the opening in the handle layer.

During the etching, the SOI wafer is mounted so that the etchant cannot contact the outward-facing surface of the device layer, on which the detector structures, including the $p n$ junctions, were previously fabricated.

Figure 3 shows a photograph of an EPI-Hi L0 detector viewed from the junction side (a) and from the ohmic side (b). The wide annular region of thin, inactive silicon in the L0 detector is needed to assure that all nuclei that have penetrated the 5-element central active regions of L1 and L2 (see Fig. 1) will have passed through the same overburden of silicon in L0, allowing the incident energies of these particles to be derived. The L1 detector looks very similar to L0, but lacks this thin, wide, inactive periphery and thus can have a smaller overall diameter (see McComas et al. 2016, Fig. 30c).

\section{Thickness characteristics}

In order to understand the thickness characteristics of the completed detectors, the thin membrane portions of the detectors were mapped using an alpha-particle energy-loss technique, which is described in Appendix A. The left panel of Fig. 4 shows a contour plot of thicknesses over the full $\sim 3.4 \mathrm{~cm}$ diameter of an L0 detector's thin silicon membrane. Measured values are dominated by the silicon, but also include contributions from the aluminum metallization and an insulating $\mathrm{SiO}_{2}$ layer overlying it. The presence of the $\mathrm{SiO}_{2}$ only outside of the active area results in the difference of mean thickness between active and inactive areas and in the modification of the contours near the boundary. The right panel of the figure shows a histogram of thickness values for the entire measured area (unfilled) and restricted to the active area (filled). The extra material outside the active area increases the thickness of this region by up to $\sim 1 \mu \mathrm{m}$ silicon equivalent.

The thickness maps of the active region were obtained from measurements made using an array of points distributed approximately uniformly over its area. From those points, we have calculated a thickness mean and standard deviation. These are plotted as the circular points in Fig. 5. Open circles indicate detectors fabricated by Micron Semiconductor while filled points indicate the prototype detectors produced by the LBNL-Caltech collaboration. Data points for thin detectors of the two types developed for EPI-Hi are shown: L0 with a thickness of $12 \mu \mathrm{m}$ and L1 with a thickness of $25 \mu \mathrm{m}$. In Fig. 5, the points corresponding to the two types are readily distinguishable because they cluster tightly near the two vertical dashed lines indicating the nominal silicon membrane thicknesses. This clustering provides a good indication of the capability of the SOI-based process for reliably yielding detectors of the desired thickness.

In contrast, Fig. 5 also shows, with $\times$ symbols, measurements of detectors that we previously flew in the Low Energy Telescope instrument (Mewaldt et al. 2008) on NASA's STEREO mission. The STEREO LET detectors had active areas of $2 \mathrm{~cm}^{2}$ and target thicknesses specified to be in the range 18-22 $\mu \mathrm{m}$. These detectors were fabricated from conventional silicon wafers (not SOI) that were lapped and polished to reduce their thickness to the desired range. As seen in the figure, nearly all of the detectors that were ultimately flown are thicker than the specified range, some significantly thicker. This is due to the fact that silicon wafers with thicknesses $\lesssim 20 \mu \mathrm{m}$ are extremely fragile, 

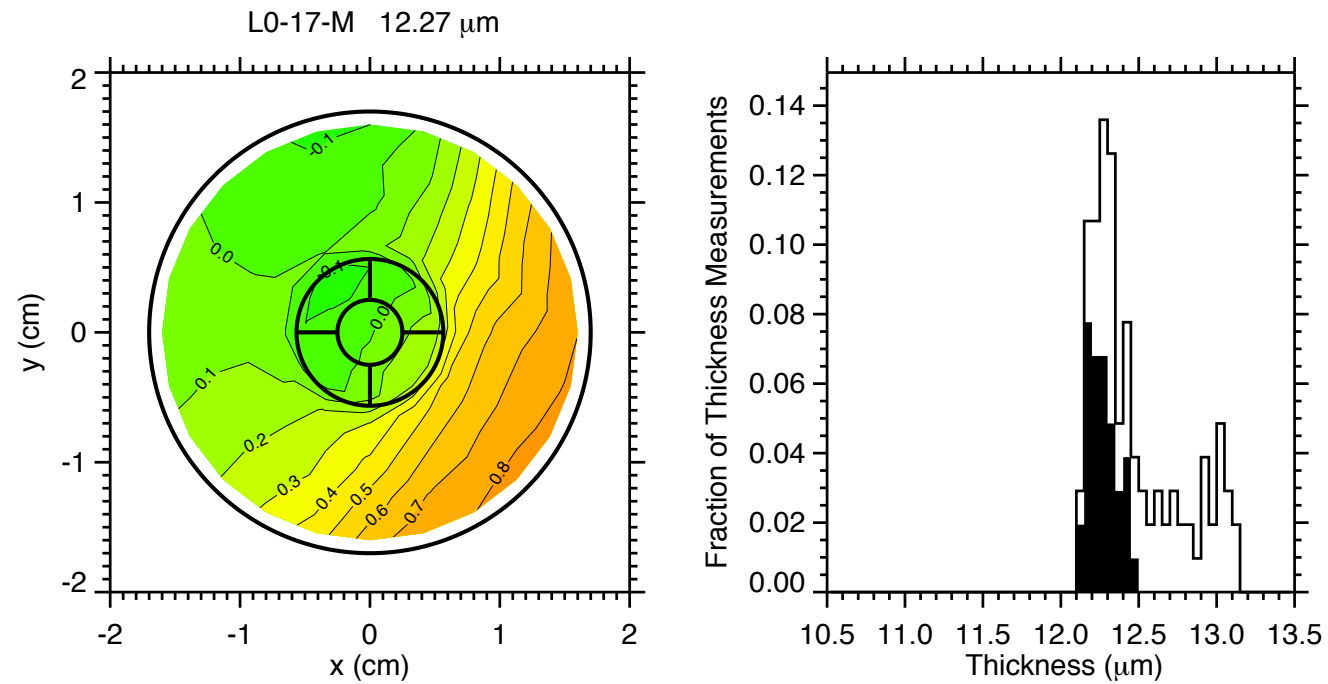

Fig. 4. Thickness measurements made over the thin area of an L0 detector. Left: contour map of thickness deviations from the value $12.27 \mu \mathrm{m}$ at the central position. Right: histograms of thickness measurements in the central active area (filled) and over the full thin area (unfilled). The distribution of measurement locations across the membrane is shown in Fig. A.1.

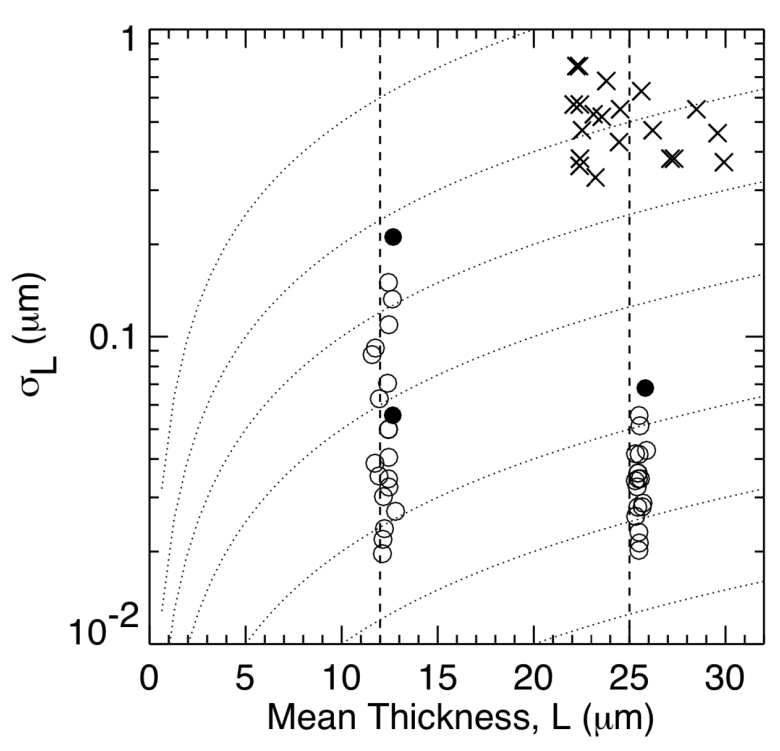

Fig. 5. Thickness, $L$, and rms thickness variation, $\sigma_{L}$, for various detectors, as follows: filled circles-prototype detectors made from SOI wafers for PSP EPI-Hi by the LBNL-Caltech collaboration; unfilled circles-detectors made from SOI wafers for PSP EPI-Hi by Micron Semiconductor; crosses-detectors made from conventional wafers for STEREO LET (Mewaldt et al. 2008) by Micron Semiconductor. The light, dotted lines indicate curves of constant $\sigma_{L} / L$ with values (top to bottom) of $5,2,1,0.5,0.2,0.1$, and $0.05 \%$.

and the manufacturing yield of such wafers was very low. Furthermore, they are very susceptible to breakage during detector manufacturing and testing.

\section{Detector performance}

\subsection{DC electrical characteristics}

Measurements of detector capacitance $(C)$ versus applied bias $(V)$ were made in order to determine the full-depletion voltage $\left(V_{\mathrm{d}}\right)$ from $C V$ curves. Typical values obtained were in the range $1.5 \pm 0.3 \mathrm{~V}$ for both the L0 and the L1 detectors. The pulse heights obtained from alpha particles penetrating these detectors, as determined by looking at oscilloscope traces of output charge signals processed with a charge-sensitive amplifier and shaped with a time constant of a few $\mu$ s, were indistinguishable for applied biases over the entire range studied $(0-30 \mathrm{~V})$.

Figure 6 shows plots of leakage current $(I)$ versus applied bias $(V)$ for typical L0 and L1 detectors at room temperature. For these measurements, the ohmic side of the detector was biased and the five main detector segments and also the annular area surrounding them were each individually held at a virtual ground by a precision op-amp used for processing the current signal. The plots show curves for each of the five segments, all of which have nearly the same current.

It is worth noting that if the outer annular region is allowed to float rather than being held at the same potential as the center segments, the currents measured on the four annular quadrant segments are much higher than the values plotted in the figure, but the current on the central bull's eye segment is essentially unchanged. Apparently, the current from the outer region is diverted to the adjacent four segments with almost none of it reaching the bull's eye segment.

Comparing the L0 and L1 IV curves in Fig. 6, it can be seen that the currents for these two detector types are essentially identical, in spite of the factor $\sim 2$ difference in the detector thicknesses. This suggests that the currents are dominated by surface effects, with negligible contribution from currents generated in the bulk volume of the silicon. This conclusion is supported by a comparison with currents observed in the L2 detectors (see Fig. 1), which have the same area and arrangement of five central segments as L0 and L1, but are $500 \mu \mathrm{m}$ thick and made from conventional silicon wafers, not from SOI. For L2, the segment currents are $\sim 400 \mathrm{nA}$, which is just $2 \times$ the corresponding L1 currents, not $20 \times$ as would be expected if the currents scaled as the active volume. This comparison provides only a qualitative indication because different silicon crystals were used for the two types of detectors.

\subsection{Energetic particle response}

The first few years of the PSP mission occurred at the minimum phase of the $\sim 11-y r$ solar activity cycle and energetic particle activity was very low. However, at energies close to 


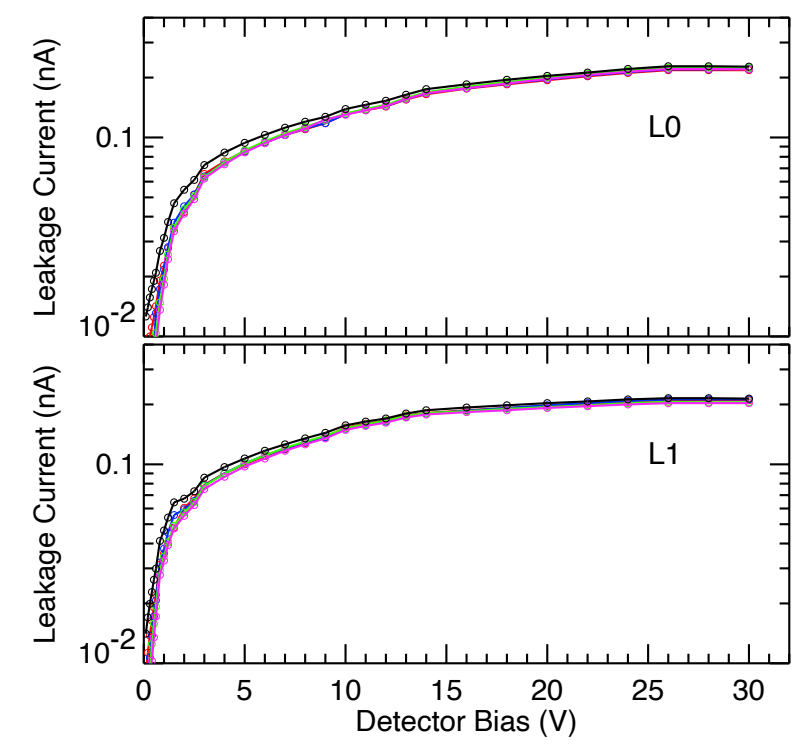

Fig. 6. Measurements of the leakage current for each of the five segments of two detectors: top-L0 detector (12 $\mu \mathrm{m}$ active thickness), bottom-L1 detector ( $25 \mu \mathrm{m}$ active thickness). The curves for the individual segments, which are shown in different colors, are difficult to distinguish because the segments have very similar currents.

$1 \mathrm{MeV} \mathrm{nuc}^{-1}$, a number of small events associated with particle acceleration near solar active regions or interplanetary stream interaction regions provided energetic $\mathrm{H}$ and $\mathrm{He}$ nuclei that were measured using the thin detectors (McComas et al. 2019; Cohen et al. 2020; Joyce et al. 2020; Leske et al. 2020; Wiedenbeck et al. 2020; Schwadron et al. 2020). The left panel of Fig. 7 shows examples of data accumulated from one of the apertures over a number of events. These measurements are compared with expected response tracks (black lines) derived using the Geant4 (GEometry ANd Tracking) simulation package (Allison et al. 2016). Such comparisons were used to fine-tune the average values of detector and dead-layer thicknesses used in the analysis.

The data in Fig. 7 were restricted to particles that triggered L0 and L1 detector segments that are vertically aligned (see LOB and L1B in Fig. 1), thereby selecting particles incident at relatively small angles, $\theta$, from the telescope axis. This population of particles has a small spread in $\sec \theta$, which yields the best charge and mass resolution. The right panel of Fig. 7 shows the He mass histogram from the time period 20-21 Apr 2020 (day of year 110-111) when ${ }^{3} \mathrm{He}$-rich solar energetic particles were detected (Wiedenbeck et al. 2020). Fitting a Gaussian to the central part of the ${ }^{4} \mathrm{He}$ peak (red curve) yields a standard deviation of $0.20 \mathrm{amu}$. We have found empirically that the smallest ${ }^{3} \mathrm{He} /{ }^{4} \mathrm{He}$ ratio that can reliably be detected at energies of a few $\mathrm{MeV}_{\text {nuc }}{ }^{-1}$ in $\mathrm{d} E / \mathrm{d} x$ versus $E$ instruments using silicon detectors is limited by tails on the low side of the ${ }^{4} \mathrm{He}$ mass distribution ${ }^{2}$ to values of a few percent. Thus, further reduction of the standard deviation of the central Gaussian part of the peak would not help with the measurement of lower ratios. Non-aligned L0-L1 segment combinations are used in measuring angular distributions of heavy element fluxes, but are not suitable for resolving isotopes due to the range of $\sec \theta$ values that they encompass.

Over the first two years of the PSP mission, EPI-Hi did not observe any events with intensities of $Z>2$ nuclei high enough

2 Low-side tails are primarily due to channeling effects in the crystalline silicon. to adequately study the instrument response to these heavy ions. Thus, we have relied on measurements of accelerator beams to characterize the heavy element response of the thin detectors (Wiedenbeck et al. 2017). Figure 8 shows measurements made using a simple three-detector stack (left panel) read out with laboratory electronics at the LBNL 88-inch cyclotron. For this test, a "cocktail beam" containing a number of different ions with $16 \mathrm{MeV} \mathrm{nuc}^{-1}$ energy and a ratio of mass to ionic charge of $\sim 2.9$ was used. In addition, we did a test using a beam of low-energy ${ }^{4} \mathrm{He}$ nuclei from a collimated alpha-particle source. These alphas are sufficiently energetic to penetrate the $12 \mu \mathrm{m}$ detector and deposit their residual energy in the following $25 \mu \mathrm{m}$ detector.

In order to populate response tracks using the monoenergetic particle populations from the accelerator or the radioactive source, we inserted an "energy spreader" in front of the detector stack. The spreader was made by stacking several layers of a thin, open-weave fabric mesh having fibers with circular cross sections. The wide range of thicknesses sampled by the particles spread out their energies. Since the resulting energy distribution was far from uniform, we sparsified the dataset to produce a more uniform distribution of energies deposited in the stopping detector. This sparsification procedure is responsible for some of the artifacts seen in Fig. 8. Others are due to particles entering or exiting the active area of detectors through the edges, to pulse pile-up in the electronics, and to channeling in the crystalline silicon.

In Fig. 8 we also show (dashed red lines) the locations of the response tracks expected from Geant 4 simulations ${ }^{3}$. The most notable discrepancy between the measured and calculated tracks is apparent in the plot of $E 1$ versus $E 2$ (top right panel) where the lowest energy portions of some measured element tracks have lower $E 1$ values than predicted from the simulation. It is well established that the mean ionic charge of heavy nuclei traversing material at low velocities is less than the nuclear charge due to the attachment of electrons from the stopping medium (e.g., Ahlen 1980). These effects, which are included among the physics processes accounted for in the Geant 4 calculation, provide much better agreement than neglecting them entirely, but clearly further improvement should be possible. Additional discrepancies, which require further investigation, can be seen for some elements in the plot of $E 2$ versus $E 3$ (bottom right panel) at the highest $E 3$ values. Once a sizable sample of heavy-ion events has been accumulated by the EPI-Hi instrument, we will be able to use those data to accurately determine the track locations. That calibration is important for onboard classification of detected heavy ions.

Wiedenbeck et al. (2017) show additional examples of the thin detector response to energetic nuclei at energies of a few $\mathrm{MeV} \mathrm{nuc}^{-1}$ from radioactive sources and from a test at the Texas A\&M University cyclotron. Since there is minimal variation of incidence angles $(\theta)$ among the particles in the accelerator beam, the charge resolution measured in those tests provides a lower limit on the resolution that can be expected in flight. There, the resolution will typically be dominated by the spread in $\sec \theta$ values. McComas et al. (2016) show some simulation results illustrating track separations expected when measuring heavy nuclei with isotropically distributed incidence angles.

\section{Environmental tests}

In order to assure that the thin detectors would survive the launch of the PSP spacecraft on a Delta IV-Heavy launch vehicle and

3 The Geant4 emstandard_opt3 physics option was used. 

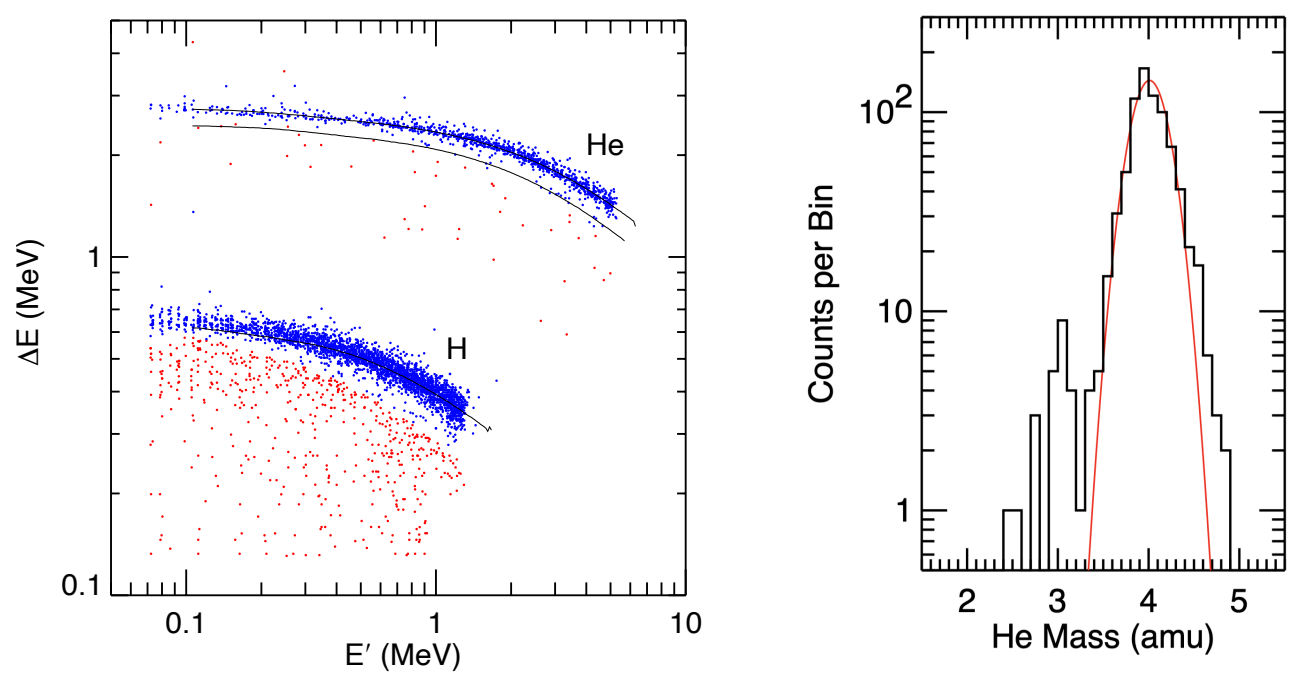

Fig. 7. Left: energy loss $(\Delta E)$ in the L0A detector versus residual energy $\left(E^{\prime}\right)$ deposited in L1A for energetic $\mathrm{H}$ and He nuclei collected by the EPI-Hi instrument during the early orbits of PSP. These data include particles incident through the A-end (generally sunward viewing end) of the LET1 telescope. The blue points indicate particles identified as $\mathrm{H}$ or He onboard; the red points indicate background events identified as not being consistent with any element. The region with a reduced density of points immediately below the $\mathrm{H}$ track is due to sampling of different regions of the plot with different efficiencies. Black lines show Geant 4 calculations of the expected $\mathrm{H},{ }^{3} \mathrm{He}$, and ${ }^{4} \mathrm{He}$ track locations. Right: He mass histogram from a two-day time period identified by Wiedenbeck et al. (2020) as having high values of ${ }^{3} \mathrm{He} /{ }^{4} \mathrm{He}(\mathrm{a}$ few $\%$ or greater, as compared to $\sim 0.04 \%$ in the solar wind). These He events were selected to have total energy greater than $4.8 \mathrm{MeV}\left(E / M>1.2 \mathrm{MeV}\right.$ nuc ${ }^{-1}$ for $\left.{ }^{4} \mathrm{He}\right)$. Red line shows Gaussian fit to the central part of the ${ }^{4} \mathrm{He}$ peak, which yields a mass resolution of $0.20 \mathrm{amu}$.

perform as required in space, environmental tests were carried out at the individual detector level, in the assembled EPI-Hi instrument, and on the PSP spacecraft. Most of the tests were typical of those for many space launches. Here we briefly discuss a few aspects specific to the unique characteristics of this detector design and this mission.

\subsection{Acoustics and vibration}

The large ratio between the diameter of the thin area of crystalline silicon and its thickness, nearly 3000:1 for the L0 detectors, was regarded as a potential risk for mechanical damage in the acoustic and vibration environment of the launch. The concern over possible damage due to acoustics was heightened due to Caltech-JPL experience during the testing for the STEREO LET instrument (Mewaldt et al. 2008), which included silicon detectors with a thickness of $\sim 25 \mu \mathrm{m}$ and a diameter of $\sim 1.6 \mathrm{~cm}$ (ratio 640:1). A significant fraction of those detectors broke during acoustic testing. Microscopic inspection of failed detectors showed that cracks in the silicon usually started at locations along the edge where there had been chipping of the silicon, probably related to the dicing of the detectors out of the silicon wafer with a diamond saw. The STEREO LET detectors were fabricated from conventional silicon wafers that had been lapped and polished down to the desired $\sim 25 \mu \mathrm{m}$ thickness, not from SOI wafers. Flexing of the thin material in the region of the edge chipping increases the stress and causes a crack to propagate from the location of the chipping.

Unlike our STEREO LET experience, none of the PSP EPI-Hi detectors were damaged in acoustics or vibration testing. We suggest the difference is attributable to the fact that the thin device layer of the SOI-based detectors is supported over a width of at least several $\mathrm{mm}$ by the bonding to the thick handle wafer (see Fig. 3b), which greatly reduces the flexing that is possible near the edge.

Vibration could, in principle, also cause membrane flexing, but for these detectors the mass of thin silicon being accelerated is so small $(<30 \mathrm{mg})$ that the force tending to flex the membrane is much less than in acoustics. No detector damage occurred as a result of the vibration tests.

\subsection{Radiation}

As an instrument designed to measure energetic nuclei down to $\sim 1 \mathrm{MeV}$ nuc $^{-1}$, EPI-Hi has negligible radiation shielding to protect the thin silicon detectors, which reside at the front of the LET telescopes behind Kapton windows that are only a few $\mu \mathrm{m}$ thick (Wiedenbeck et al. 2017). Furthermore, the intensities of energetic particle events close to the Sun are expected to be higher than values commonly experienced near Earth, possibly increasing with decreasing heliocentric radius, $r$, as $1 / r^{2}$ or faster. The EPI-Hi instrument needs to continue to perform well even after exposure to this radiation environment (Lario \& Decker 2011b,a) over the seven-year prime mission of PSP.

Total-dose tests were carried out at JPL using ${ }^{60} \mathrm{Co}$ gamma rays up to a dose of $10 \mathrm{Mrad}$ and a displacement damage test at the University of California Davis cyclotron was performed using a total fluence of $10^{13} / \mathrm{cm}^{2}$ of $67.5 \mathrm{MeV}$ protons. The significantly increased leakage currents measured following these radiation exposures were still at acceptable levels. The detector energy resolution, measured using an alpha-particle source, was not significantly degraded ${ }^{4}$.

\footnotetext{
4 In one prototype detector design, the contact to the ohmic surface was made through the bulk of the inactive silicon near the edge of the device layer. The displacement damage test increased the resistivity of this $n$ type silicon and made the $R C$ time constant due to the combination of this resistive contact and the large detector capacitance comparable to the few $\mu$ s of the pulse-shaping circuit and reduced the measured pulse height. However, paralleling this contact with a conventional wire-bond contact directly to the ohmic surface restored the pre-exposure pulse heights. On all of the flight detectors, wire bonds to the ohmic surface were used.
} 

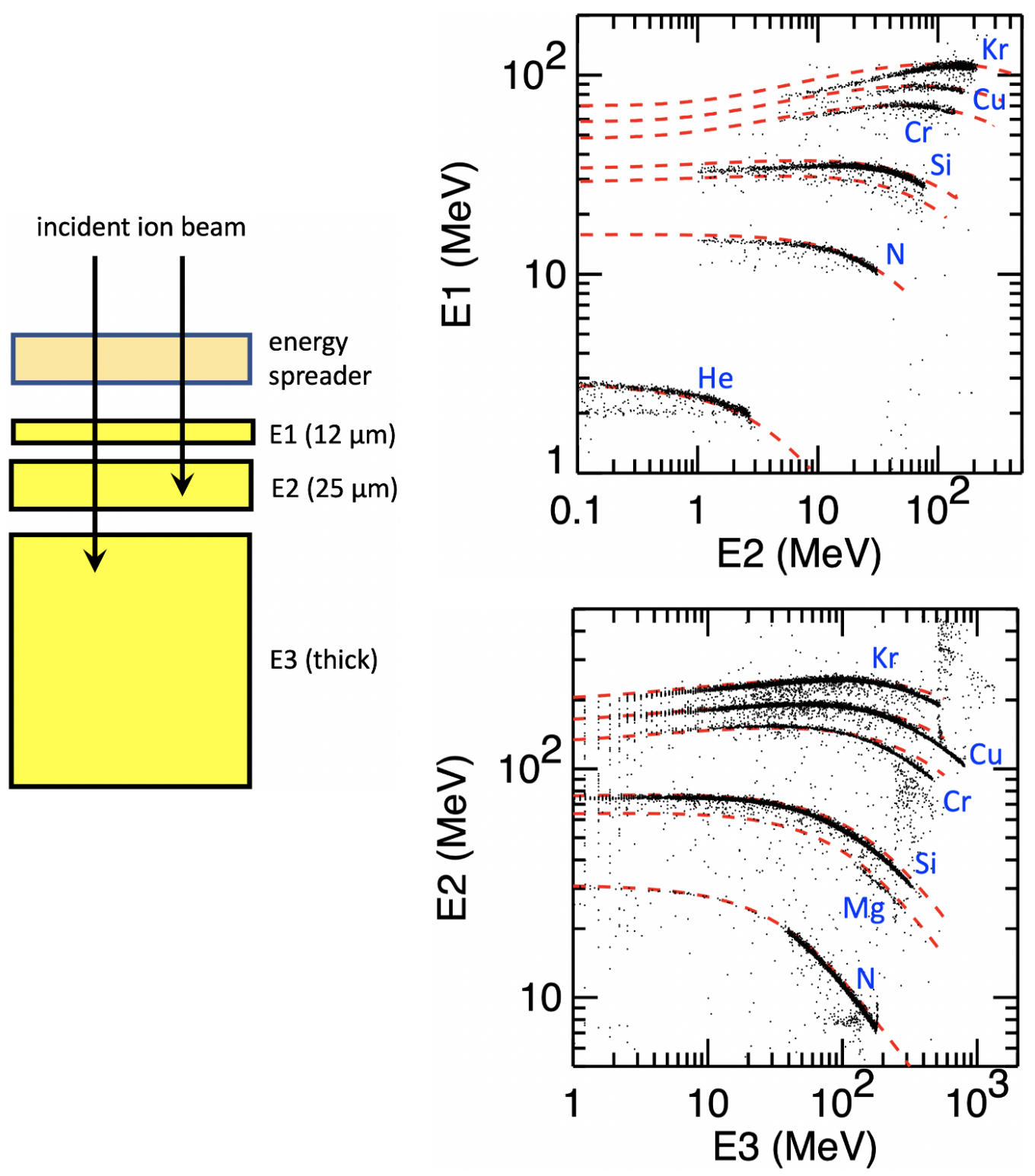

Fig. 8. Response of a simple detector telescope to heavy ions compared with a Geant4 simulation. Data were collected at the LBNL 88 -inch cyclotron using a cocktail beam containing a variety of $16 \mathrm{MeV} \mathrm{nuc}{ }^{-1}$ ions with $M / Q \simeq 2.9$ including ${ }_{7}^{14} \mathrm{~N}^{+5},{ }_{12}^{26} \mathrm{Mg}^{+9},{ }_{14}^{29} \mathrm{Si}^{+10},{ }_{24}^{54} \mathrm{Cr}^{+19},{ }_{29}^{63} \mathrm{Cu}^{+22}$, and ${ }_{36}^{78} \mathrm{Kr}^{+27}$. Also shown is the response to ${ }_{2}^{4} \mathrm{He}$ obtained from a collimated alpha-particle source. The measured response tracks (black) are compared with Geant4 simulations (dashed red lines) calculated assuming the same detector and dead-layer thicknesses used for calculating the tracks in Fig. 7. Left: schematic illustration of the telescope configuration consisting of two thin detectors followed by a thick detector. In front of the telescope was placed an "energy spreader" consisting of multiple layers of thin fabric mesh that served to provide a wide distribution of particle ranges entering the telescope. Top right: $\Delta E$ versus $E^{\prime}$ tracks for particles stopping in the E2 detector. Bottom right: response tracks for particles stopping in the E3 detector.

\subsection{Dust impacts}

A population of microscopic dust particles produced by multiple impacts of larger bodies pervades much of the inner heliosphere (Szalay et al. 2020, and references therein). At heliocentric distances that will be traversed by PSP, velocities of many such particles relative to the spacecraft will exceed several $100 \mathrm{~km} \mathrm{~s}^{-1}$. Impacts of these small, fast particles have the potential to penetrate the LET windows and impact the thin LO detectors behind them. Therefore, tests were carried out at dust accelerators at the University of Colorado and at Heidelberg, Germany. The highest dust velocities and kinetic energies available from the accelerators were significantly less than could be encountered in flight, but did provide some useful insights into the effects that fast dust is likely to have. Dust particles were found to be able to penetrate the thin windows and produce pinholes comparable to the size of the dust particle, but the windows did not rupture. The LET design (Wiedenbeck et al. 2017) uses three windows spaced several mm apart (Fig. 1) to reduce the chance of light penetrating to the detectors ${ }^{5}$. Dust impacts on an L0 detector produced impact craters, but did not penetrate or rupture the detector. Impacts were found to cause significant and long-lasting increases in leakage current, but only the segment that was directly hit was

\footnotetext{
5 Near the Sun, EPI-Hi is always in the shadow of the PSP heat shield, but it is still possible for some scattered light from the solar corona to reach the instrument.
} 
affected. Successive impacts on the same segment had cumulative effects, producing an irregular stair-step increase of leakage current versus time.

Over the first six orbits of PSP, which reached heliocentric distances near 0.095 au, no leakage-current increases thought to be associated with dust impacts were observed. However, other PSP instruments have provided definitive evidence of dust impacts on the spacecraft, including one that punctured a very thin collimator foil on the IS $\odot$ IS Low-energy Energetic Particles Instrument (EPI-Lo; McComas et al. 2016; Szalay et al. 2020).

\subsection{Temperature}

Parker Solar Probe was designed to allow instruments such as EPI-Hi to operate near room temperature (Fox et al. 2016) in spite of the intense heat input on the sunward-facing surface of the spacecraft. Thus, specifications called for the detectors to be tested between $-40{ }^{\circ} \mathrm{C}$ and $+40{ }^{\circ} \mathrm{C}$ for operation, similar to many near-Earth spacecraft, and between $-60^{\circ} \mathrm{C}$ and $+60{ }^{\circ} \mathrm{C}$ for survival. These requirements were met without difficulty. The EPI-Hi thermal design passively cools the detector telescopes by radiation to space. Throughout the orbit, the detector temperatures have been stable around $-22^{\circ} \mathrm{C}$, which is conducive to low-noise operation.

\section{Future prospects}

The SOI-based approach for producing thin, uniform silicon detectors can be extended to yield even thinner devices. In one of our earliest efforts to make detectors from SOI wafers, Caltech thinned some SOI having device layers as thin as $5 \mu \mathrm{m}$, diced them into chips with thin areas $\sim 1 \mathrm{~cm}^{2}$, and had AmetekOrtec process those chips to make surface-barrier detectors. This detector type, which was commonly used prior to the widespread availability of ion-implanted detectors, employs a Schottkybarrier diode formed between the silicon and a metal evaporated on its surface. Detectors produced in this way detected alpha particles, but accelerator tests showed that under certain circumstances charge-multiplication effects (Tsyganov et al. 1996, and references therein) prevented reliable measurements of the energies deposited by heavier ions. These devices also had higher leakage currents than the ion-implanted detectors. The surface-barrier technology was rejected for further development, but these detectors did allow us to successfully demonstrate a process for SOI thinning.

More recently, the LBNL-Caltech collaboration made SOI-based, ion-implanted detectors with active areas as large as $4 \mathrm{~cm}^{2}$ and silicon membranes as thin as $5 \mu \mathrm{m}$. It was not possible to pursue testing of those devices because of the demands on Caltech personnel to develop the EPI-Hi instrument for PSP.

We anticipate that work in the near future will lead to SOI-based, ion-implanted detectors at least as thin as $5 \mu \mathrm{m}$ that are suitable for spaceflight and laboratory applications.

Larger active areas are also of interest for future detector applications. Although the EPI-Hi L0 detector's active area was only $1 \mathrm{~cm}^{2}$, that active region was located at the center of a $12 \mu \mathrm{m}$ silicon membrane having an area of $\sim 9 \mathrm{~cm}^{2}$ and the processing included fabrication of electrical traces that extend from the active area to the edge. Thus, it appears that it should be possible to produce detectors having at least this area and thickness. Since the capacitance per unit area of thin detectors is high ( $\sim 900 \mathrm{pF} \mathrm{cm}^{-2}$ for a $12 \mu$ m detector), we would expect that large-area, thin detectors will be highly segmented. Thin silicon strip or pixel detectors seem like natural applications meriting further development.

Acknowledgements. We thank W. C. Tang for suggesting the use of SOI wafers as the basis for thin detector fabrication, B. Eyre for research on optimizing etching processes, J. Klemic (deceased) for developing the etching process used for thinning the detectors processed at LBNL, and B. D. Milliken for pioneering the technique used for thickness mapping. We thank S. Holland for useful discussions and S. M. Krimigis provided helpful comments about the thin silicon detectors used on Voyager. Important contributions to the success of the IS๑IS EPI-Hi instrument were made by numerous other scientists, engineers, and technicians. Particular thanks are due to G. Dirks, D. H. Do, J. J. Hanley, L. Hernandez, T. L. Johnson, S. Lopez, H. Miyasaka, G. Riggins, B. Rodriguez, K. Simms, and M. L. White. We gratefully acknowledge the test support provided by Michigan State University's National Superconducting Cyclotron Laboratory, Texas A\&M University's Cyclotron Institute, and the Lawrence Berkeley National Laboratory's 88-inch Cyclotron Laboratory. This work was supported, in part, by NASA's Parker Solar Probe Mission, contract NNN06AA01C. Parker Solar Probe was designed, built, and is now operated by the Johns Hopkins Applied Physics Laboratory as part of NASA's Living with a Star (LWS) program. Support from the LWS management and technical team has played a critical role in the success of the Parker Solar Probe mission. Earlier detector development work at JPL, Caltech, and LBNL was supported by several NASA grants. The IS $\odot$ IS data and visualization tools are available to the community at https://spacephysics.princeton.edu/ missions-instruments/isois; data are also available via the NASA Space Physics Data Facility (https://spdf.gsfc.nasa.gov/).

\section{References}

Ahlen, S. P. 1980, Rev. Mod. Phys., 52, 121

Allison, J., Amako, K., Apostolakis, J., et al. 2016, Nucl. Instrum. Methods Phys. Res. A, 835, 186

Cohen, C. M. S., Christian, E. R., Cummings, A. C., et al. 2020, ApJS, 246, 20

Fox, N. J., Velli, M. C., Bale, S. D., et al. 2016, Space Sci. Rev., 204, 7

Gosele, U., \& Tong, Q.-Y. 1998, Annu. Rev. Mater. Res., 28, 215

Hamilton, D. C., Gloeckler, G., Krimigis, S. M., \& Lanzerotti, L. J. 1981, J. Geophys. Res., 86, 8301

Hamilton, D. C., Brown, D. C., Gloeckler, G., \& Axford, W. I. 1983, J. Geophys. Res., 88, 8905

Hill, M. E., Mitchell, D. G., Andrews, G. B., et al. 2017, J. Geophys. Res. Space Phys., 122, 1513

Joyce, C. J., McComas, D. J., Christian, E. R., et al. 2020, ApJS, 246, 41

Kovacs, G. T., Maluf, N. I., \& Petersen, K. E. 1998, Proc. IEEE, 86, 1536

Krimigis, S. M., Armstrong, T. P., Axford, W. I., et al. 1977, Space Sci. Rev., 21, 329

Lario, D., \& Decker, R. B. 2011a, Space Weather, 9, S12002

Lario, D., \& Decker, R. B. 2011b, Space Weather, 9, S11003

Leske, R. A., Christian, E. R., Cohen, C. M. S., et al. 2020, ApJS, 246, 35

Mason, G. M., Gold, R. E., Krimigis, S. M., et al. 1998, Space Sci. Rev., 86, 409

McComas, D. J., Alexander, N., Angold, N., et al. 2016, Space Sci. Rev., 204, 187

McComas, D. J., Christian, E. R., Cohen, C. M. S., et al. 2019, Nature, 576, 223

Mewaldt, R. A., Cohen, C. M. S., Cook, W. R., et al. 2008, Space Sci. Rev., 136, 285

Schwadron, N. A., Bale, S., Bonnell, J., et al. 2020, ApJS, 246, 33

Stone, E. C., Cohen, C. M. S., Cook, W. R., et al. 1998a, Space Sci. Rev., 86, 285

Stone, E. C., Cohen, C. M. S., Cook, W. R., et al. 1998b, Space Sci. Rev., 86, 357

Szalay, J. R., Pokorný, P., Bale, S. D., et al. 2020, ApJS, 246, 27

Tabata, O., Asahi, R., Hirofumi, F., Kenchi, S., \& Sugiyama, S. 1992, Sens. Actuators, 34, 51

Tsyganov, Y., Kushniruk, W., \& Polyakov, A. 1996, IEEE Transac. Nucl. Sci., 43, 2496

von Rosenvinge, T. T., McDonald, F. B., Trainor, J. H., Van Hollebeke, M. A. I., \& Fisk, L. A. 1978, IEEE Transac. Geosci. Electron., 16, 208

von Rosenvinge, T. T., Barbier, L. M., Karsch, J., et al. 1995, Space Sci. Rev., 71, 155

Wiedenbeck, M. E., Angold, N. G., Birdwell, B., et al. 2017, Int. Cosmic Ray Conf., 301, 16

Wiedenbeck, M. E., Bučík, R., Mason, G. M., et al. 2020, ApJS, 246, 42

Wolf, S., \& Tauber, R. N. 1998, Silicon Processing for the VLSI Era, Process Technology (Sunset Beach, CA: Lattic Press), 1 


\section{Appendix A: Thickness measurements}

Maps of the spatial dependence of thin-detector thicknesses were made to determine the mean thickness and thickness variation, as shown in Fig. 4. This appendix describes the non-contact method that was used to obtain calibrated measurements with a typical precision better than $0.1 \mu \mathrm{m}$. The general approach used was to allow a collimated beam of alpha particles from a radioactive source $\left({ }^{244} \mathrm{Cm}\right.$ or $\left.{ }^{228} \mathrm{Th}\right)$ to penetrate the thin silicon membrane and then stop in a small silicon detector on the other side. The mean residual energy deposited in that detector is directly related to the thickness penetrated and can be calibrated by performing the corresponding measurement on samples of known thickness.

The left panel of Fig. A.1 is a schematic illustration of the setup. The alpha source and the silicon detector were mounted a few $\mathrm{cm}$ apart. A pair of sheet metal plates, each having one small hole (typically $2 \mathrm{~mm}$ diameter), were placed between the source and the detector, as shown. These parts were all held fixed relative to one another. The sample to be measured (detector or calibration foil) was inserted between the two collimator plates such that it could be moved in two dimensions perpendicular to the alpha-particle beam using a pair of computer-controlled translation stages. For the measurements, this assembly was installed in a vacuum system.

The sample being measured was successively moved to each of the points at which thickness measurements were desired (top right panel) and a spectrum of residual energies deposited in the detector was recorded. In the active region (red points), the point spacing was chosen to be comparable to the radius of the alpha particle beam. In the inactive outer region (blue points), a wider spacing was used to reduce the mapping time. Fits of Gaussians to the central portions of each of the residual-energy peaks yielded a set of means, standard deviations, and areas (numbers of counts) for the Gaussians. For many of the samples we studied, the variation of the mean over the area of the sample was smaller than the standard deviation. So, thousands of counts per point were typically used so that the location of the mean could be determined with the required precision.

In terms of $\mathrm{g} \mathrm{cm}^{-2}$, a single sheet of household aluminum foil (not "heavy duty") is equivalent to $\sim 18 \mu \mathrm{m}$ of silicon and in terms of the specific ionization, these two adjacent elements are very similar. So, for calibrating the measurements of the L0 $(\sim 12 \mu \mathrm{m})$ and L1 $(\sim 25 \mu \mathrm{m})$ detectors we chose to use 0,1 , and 2 layers of household aluminum foil. To obtain a precise value of the foil's areal density, a full roll was unwound from its cardboard tube and weighed. Dividing the mass by the measured area of the foil yielded its mean areal density. Numerous samples were taken from various locations distributed over the foil to check the uniformity of the roll. We found the foil to be extremely uniform in thickness throughout the entire roll. Measurements of residual energies with each of the calibration samples in the alpha-particle beam were made for comparison with the silicon measurements.
After deriving the $\mathrm{g} \mathrm{cm}^{-2}$ of aluminum required to produce the measured residual energies, we converted to equivalent $\mathrm{g} \mathrm{cm}^{-2}$ of silicon using the ratio between the number of electrons per $\mathrm{cm}^{3}$ in aluminum and silicon. Finally, conversion from $\mathrm{g} \mathrm{cm}^{-2}$ of silicon to thickness of silicon was done using the standard silicon density of $2.33 \mathrm{~g} \mathrm{~cm}^{-3}$. The small difference in electron binding energy between aluminum and silicon was neglected.

Rather than simply doing a linear interpolation between the calibration points, we used a modified interpolation procedure that takes advantage of the reasonably well known range-energy relation for alpha particles in silicon. This was motivated by the fact that silicon-equivalent thicknesses of 0,1 , and 2 layers of aluminum foil are fairly widely spaced, leading to inaccuracy in the linear interpolation. Our procedure depends on the alpha-particle range-energy relation, $R_{\alpha}=\mathcal{R}\left(E_{\alpha}\right)$, and its inverse function, $E_{\alpha}=\mathcal{E}\left(R_{\alpha}\right)$.

We consider two samples with thicknesses $L_{i}$ and $L_{j}$. If alpha particles with a range $R_{\alpha}$ penetrate sample $i(j)$ and deposit residual energy $E_{i}^{\prime}\left(E_{j}^{\prime}\right)$ in the detector, the ratio of the residual energies, $\Gamma_{i j}$, is related to $R_{\alpha}, L_{i}$ and $L_{j}$ by

$\Gamma_{i j} \equiv \frac{E_{i}^{\prime}}{E_{j}^{\prime}}=\frac{\mathcal{E}\left(R_{\alpha}-L_{i}\right)}{\mathcal{E}\left(R_{\alpha}-L_{j}\right)}$.

Letting samples $i$ and $j$ be the two calibration samples, Eq. (A.1) can be used to calculate $R_{\alpha}$, which is the same for all of the measurements. Now, if the thickness $L_{k}$ of another sample is to be derived by comparing its $E_{k}^{\prime}$ with the calibration values $E_{i}^{\prime}$ and $E_{j}^{\prime}$, we just apply Eq. (A.1) to $\Gamma_{i k}$ using the same values of $L_{i}$ and $R_{\alpha}$. Since this procedure does not depend on absolute values of the residual energies, but only on ratios among them, the calculation can be carried out using measured pulse heights without needing to know the gain of the electronics (providing that nonlinearities in these measurements are either negligible or corrected for).

Besides being insensitive to the electronic gain used for the residual energy measurements, the above procedure is also insensitive to the dead layer on the $E^{\prime}$ detector and to any thin coating over the radioactive source. As long as these are the same for the calibration measurements as for measurements of the samples being studied, they do not play any role. Using the setup shown in the left panel of Fig. A.1, in which the source and detector are kept fixed relative to one another, these values remain the same for all measurements.

These measurements yield the physical thickness of the sample, not its active thickness. To date, we have treated the thin-detector dead layers as parameters that need to be adjusted to obtain the best agreement between the measured locations of $\Delta E-E^{\prime}$ tracks and locations calculated from a range-energy relation (see Fig. 7). 

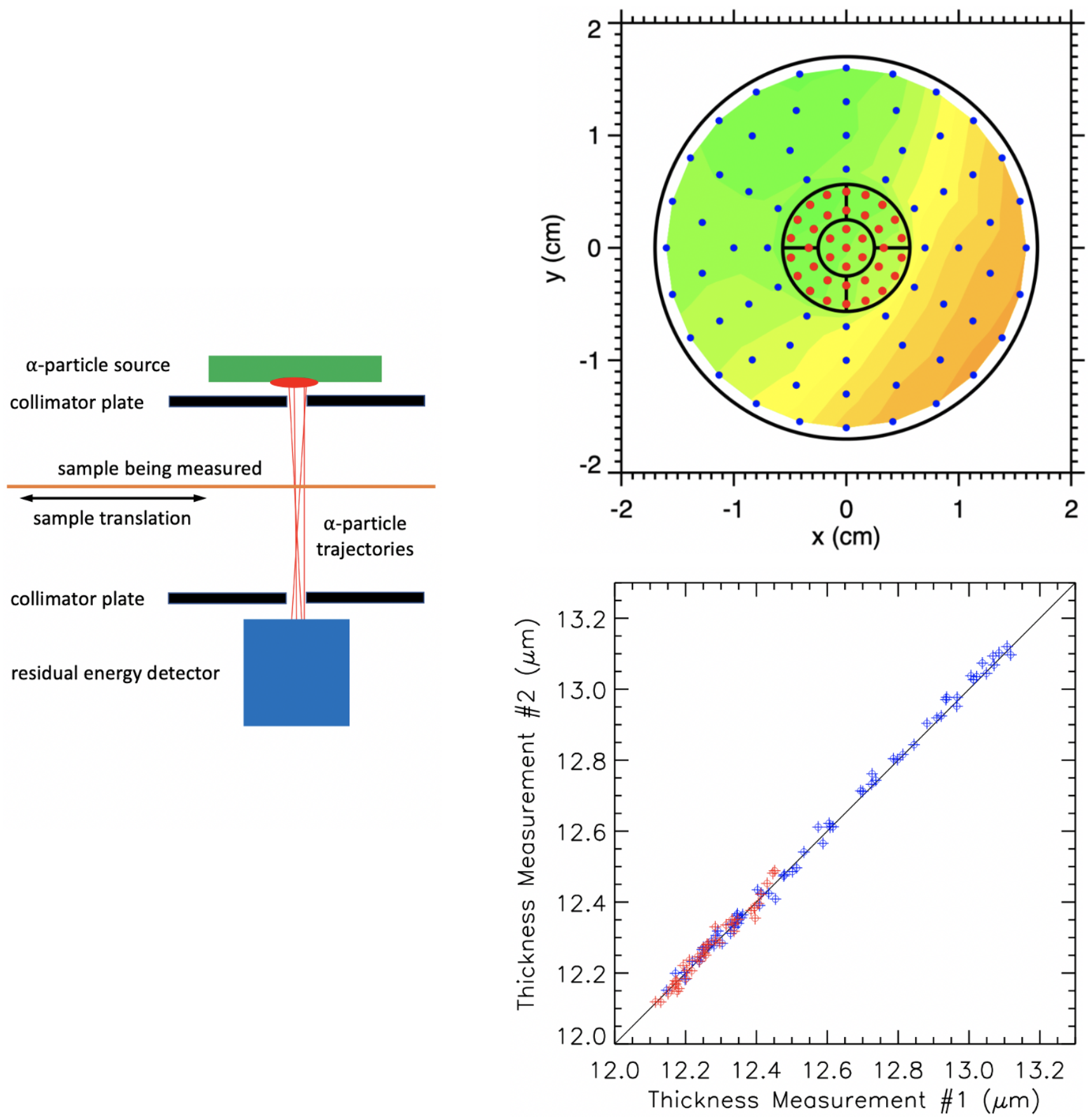

Fig. A.1. Left: schematic illustration of the setup used for mapping detector thicknesses. The locations of all parts were fixed relative to one another other than the sample being measured, which was translated in two dimensions perpendicular to the beam of $\alpha$ particles. Top right: locations at which measurements were made superimposed on the color contour map from Fig. 4 . The red points are located within the active region of the detector; the blue points are located in the inactive peripheral region. The radius of the alpha beam at the detector was comparable to the distance between adjacent points in the active region. Bottom right: comparison of thicknesses derived from two separate runs mapping the same L0 detector. The error bars were derived from the statistical uncertainties in the mean positions of the Gaussians fitted to the residual energy distributions. Red (blue) indicates active (inactive) region points, as in the top right panel. 